\title{
Mean value theorem and semigroups of operators for interval-valued functions on time scales
}

\author{
Yonghong Shen (D) \\ School of Mathematics and Statistics, Tianshui Normal University, Tianshui 741001, P.R. China
}

\begin{abstract}
In this paper, a new version of mean value theorem for interval-valued functions on time scales is established. Meantime, some basic concepts and results associated with semigroups of operators for interval-valued functions on time scales are presented. As an application of semigroups of operators, under certain conditions, we consider the initial value problem for interval-valued differential equations on time scales. Finally, two issues worthy of further discussion are presented.
\end{abstract}

Mathematics Subject Classification (2020). 49J53, 54C60, 58C06

Keywords. interval-valued functions, mean value theorem, semigroup of operators, time scales

\section{Introduction}

In 1988, the notion of time scale was introduced by Hilger [6] to unify continuous and discrete analysis. There is no doubt that the time scale calculus provide a unified framework for the study of differential equations and difference equations. In practice, many problems involve various types of uncertainty. Usually, the knowledge about the parameters of a real world system is imprecise or uncertain because, generally, it is difficult to accurately observe or measure the true value of these parameters. In these cases, the value of a parameter cannot be characterized by an ordinary real number. Accordingly, interval numbers and fuzzy numbers are two important tools to deal with these problems. In fact, interval numbers can be regarded as a special case of fuzzy numbers. Taking into account the shortcoming of the difference of fuzzy numbers, it is necessary to carry out the study of interval analysis. More importantly, interval analysis can provide important methodologies and foundations for fuzzy analysis. In 1993, Markov [8] first studied the differentiability and integrability of interval-valued functions. Later, Stefanini and Bede [11] together with Chalco-Cano et al. [3] further extended the theory of calculus of interval-valued functions. In 2013, Lupulescu [7] introduced the differentiability and integrability for interval-valued functions on time scales by using the generalized Hukuhara differentiability.

The mean value theorem for real-valued functions has important and extensive application in the classical calculus. In [8], the mean value theorem for interval-valued functions was established. Afterwards, the work was extended to the interval-valued functions on time scales by Lupulescu [7]. One purpose of this paper is to give a new version of the

Email address: shenyonghong2008@hotmail.com

Received: 09.11.2019; Accepted: 02.05.2020 
mean value theorem for interval-valued functions on time scales. In addition, semigroups of operators are very important in the study of differential equations. In 2005, the semigroups of operators on spaces of fuzzy-number-valued functions were proposed by Gal and Gal [4] and were applied to study fuzzy differential equations. Recently, Hamza and Oraby [5] developed the theory of semigroups of operators on time scales. Motivated by these works, the other purpose of the present paper is to present some basic concepts and results related to semigroups of operators for interval-valued functions on time scales.

\section{Preliminaries}

Let $\mathbb{Z}_{0}^{+}, \mathbb{R}_{0}^{+}$and $\mathbb{R}$ denote the set of all nonnegative integers, nonnegative real numbers and real numbers, respectively. Denote by $\mathcal{K}$ the set of all nonempty compact convex subsets (i.e., bounded and closed intervals) of the real line $\mathbb{R}$. For $A=\left[a^{-}, a^{+}\right], B=$ $\left[b^{-}, b^{+}\right] \in \mathcal{K}, \lambda \in \mathbb{R}$, the Minkowski addition $A+B$ and scalar multiplication $\lambda \cdot A$ (denoted by $\lambda A$ ) can be defined by

$$
A+B=\left[a^{-}, a^{+}\right]+\left[b^{-}, b^{+}\right]=\left[a^{-}+b^{-}, a^{+}+b^{+}\right]
$$

and

$$
\lambda \cdot A=\lambda A=\lambda\left[a^{-}, a^{+}\right]=\left[\min \left\{\lambda a^{-}, \lambda a^{+}\right\}, \max \left\{\lambda a^{-}, \lambda a^{+}\right\}\right] .
$$

It is well know that the addition is associative and commutative and with the neutral element $\{0\}$. Especially, if $\lambda=-1$, then the scalar multiplication gives the opposite $-A=(-1) A=\left[-a^{+},-a^{-}\right]$. However, in general, $A+(-A) \neq\{0\}$. That is to say, the opposite of $A$ is not the inverse of $A$ with respect to the Minkowski addition, unless $A$ is a singleton.

Let $A, B \in \mathcal{K}$. If there exists $C \in \mathcal{K}$ such that $A=B+C$, then $C$ is called the Hukuhara difference (or H-difference) of $A$ and $B$, and it is denoted by $C:=A \ominus B$. Note that the $\mathrm{H}$-difference is unique, but it does not always exist for any two intervals. Given two intervals $A, B \in \mathcal{K}$, it is easy to know that the H-difference $A \ominus B$ exists if and only if $\operatorname{len}(A) \geq \operatorname{len}(B)$, where $\operatorname{len}(\cdot)$ denotes the length of the interval, i.e., $\operatorname{len}(A)=a^{+}-a^{-}$. In order to overcome this shortcoming, the generalized difference is introduced as follows.

Definition 2.1 (Markov [8], Stefanini [10]). Let $A, B \in \mathcal{K}$. The generalized Hukuhara difference ( $\mathrm{gH}$-difference for short) is defined as

$$
A \ominus_{g} B=C \Leftrightarrow\left\{\begin{array}{l}
(i) A=B+C \Leftrightarrow A \ominus B=C, \\
\text { or }(i i) B=A+(-C) \Leftrightarrow B \ominus A=-C .
\end{array}\right.
$$

According to Def. 2.1, if $A=\left[a^{-}, a^{+}\right], B=\left[b^{-}, b^{+}\right] \in \mathcal{K}$, then we have

$$
\begin{aligned}
A \ominus_{g} B & =\left[a^{-}, a^{+}\right] \ominus_{g}\left[b^{-}, b^{+}\right] \\
& =\left[\min \left\{a^{-}-b^{-}, a^{+}-b^{+}\right\}, \max \left\{a^{-}-b^{-}, a^{+}-b^{+}\right\}\right] \\
& = \begin{cases}{\left[a^{-}-b^{-}, a^{+}-b^{+}\right],} & \operatorname{len}(A) \geq \operatorname{len}(B), \\
{\left[a^{+}-b^{+}, a^{-}-b^{-}\right],} & \operatorname{len}(A)<\operatorname{len}(B) .\end{cases}
\end{aligned}
$$

From $[8,10,12]$, some basic properties of gH-difference can be summarized as follows.

(i) $A \ominus_{g} A=\{0\}, A \ominus_{g}\{0\}=A,\{0\} \ominus_{g} A=-A$;

(ii) $A \ominus_{g} B=(-B) \ominus_{g}(-A)=-\left(B \ominus_{g} A\right)$;

(iii) $A \ominus_{g}(-B)=B \ominus_{g}(-A),(-A) \ominus_{g} B=(-B) \ominus_{g} A$;

(iv) $(A+B) \ominus_{g} B=A, A \ominus_{g}(A+B)=-B$;

(v) $\left(A \ominus_{g} B\right)+B=A$ if $\operatorname{len}(A) \geq \operatorname{len}(B), A+(-1)\left(A \ominus_{g} B\right)=B$ if $\operatorname{len}(A)<\operatorname{len}(B)$;

(vi) $\lambda\left(A \ominus_{g} B\right)=\lambda A \ominus_{g} \lambda B, \lambda \in \mathbb{R}$;

(vii) $(\lambda+\mu) A=\lambda A+\mu A$ if $\lambda \mu \geq 0,(\lambda+\mu) A=\lambda A \ominus_{g}(-\mu A)$ if $\lambda \mu<0$. 
Lemma 2.2. Let $A=\left[a^{-}, a^{+}\right], B=\left[b^{-}, b^{+}\right]$and $C=\left[c^{-}, c^{+}\right]$belong to $\mathcal{K}$. Then:

(i) If len $(A) \geq \operatorname{len}(C)$, then $(A+B) \ominus_{g} C=\left(A \ominus_{g} C\right)+B$;

(ii) If len $(A)<\operatorname{len}(C)$, then $(A+B) \ominus_{g} C=\left(A \ominus_{g} C\right) \ominus_{g}(-B)$.

Proof. For simplicity, we write $(A+B) \ominus_{g} C=D$, where $D=\left[d^{-}, d^{+}\right]$.

(i) If len $(A) \geq \operatorname{len}(C)$, then $(A+B) \ominus_{g} C=(A+B) \ominus C$. Using the representation of endpoints, we have

$$
\begin{aligned}
(A+B) \ominus_{g} C & =(A+B) \ominus C \\
& =\left[a^{-}+b^{-}-c^{-}, a^{+}+b^{+}-c^{+}\right] \\
& =\left[a^{-}-c^{-}, a^{+}-c^{+}\right]+\left[b^{-}, b^{+}\right] \\
& =(A \ominus C)+B \\
& =\left(A \ominus_{g} C\right)+B .
\end{aligned}
$$

(ii) If $\operatorname{len}(A)<\operatorname{len}(C)$, then $A \ominus_{g} C=-(C \ominus A)$. Therefore, we can infer from Definition 2.1 that

$$
\begin{aligned}
& \left(A \ominus_{g} C\right) \ominus_{g}(-B) \\
= & {\left[a^{+}-c^{+}, a^{-}-c^{-}\right] \ominus_{g}\left[-b^{+},-b^{-}\right] } \\
= & {\left[\min \left\{a^{+}-c^{+}+b^{+}, a^{-}-c^{-}+b^{-}\right\}, \max \left\{a^{+}-c^{+}+b^{+}, a^{-}-c^{-}+b^{-}\right\}\right] } \\
= & (A+B) \ominus_{g} C .
\end{aligned}
$$

Now we define a functional $\|\cdot\|: \mathcal{K} \rightarrow[0, \infty)$ by $\|A\|=\max \left\{\left|a^{-}\right|,\left|a^{+}\right|\right\}$for every $A=\left[a^{-}, a^{+}\right] \in \mathcal{K}$. It can easily be shown that $\|\cdot\|$ is a norm on $\mathcal{K}$, and thus the quadruple $(\mathcal{K},+, \cdot,\|\cdot\|)$ is a normed quasilinear space [9].

Given two intervals $A=\left[a^{-}, a^{+}\right], B=\left[b^{-}, b^{+}\right] \in \mathcal{K}$, the Hausdorff-Pompeiu metric between $A$ and $B$ is defined by $d_{H}(A, B)=\max \left\{\left|a^{-}-b^{-}\right|,\left|a^{+}-b^{+}\right|\right\}$. It is well known that $\left(\mathcal{K}, d_{H}\right)$ is a complete and separable metric space. Furthermore, the following relationships exist between the Hausdorff-Pompeiu metric $d_{H}$ and the norm $\|\cdot\|$ :

$$
\|A\|=d_{H}(A,\{0\}), \quad d_{H}(A, B)=\left\|A \ominus_{g} B\right\| .
$$

In addition, for all $A, B, C, D \in \mathcal{K}$, the metric $d_{H}$ has the following properties:

(i) $d_{H}(A+B, A+C)=d_{H}(B, C)$,

(ii) $d_{H}(\lambda A, \lambda, B)=|\lambda| d_{H}(A, B), \lambda \in \mathbb{R}$,

(iii) $d_{H}(A+C, B+D) \leq d_{H}(A, B)+d_{H}(C, D)$,

(iv) $d_{H}\left(A \ominus_{g} B, A \ominus_{g} C\right) \leq d_{H}(B, C)$.

Here, we briefly recall some basic notions related to the time scale. For more details, we recommend two excellent monographs $[1,2]$ written by Bohner and Peterson. A time scale $\mathbb{T}$ is a nonempty closed subset of $\mathbb{R}$. For $t \in \mathbb{T}$, the forward jump operator $\sigma$ and the back jump operator $\rho$ are defined as $\sigma(t):=\inf \{s \in \mathbb{T}: s>t\}$ and $\rho(t):=\sup \{s \in \mathbb{T}: s<t\}$, respectively. Especially, $\inf \emptyset=\sup \mathbb{T}, \sup \emptyset=\inf \mathbb{T}$.

A point $t \in \mathbb{T}$ is said to be right-scattered, right-dense, left-scattered and left-dense if $\sigma(t)>t, \sigma(t)=t, \rho(t)<t$ and $\rho(t)=t$, respectively. Given a time scale $\mathbb{T}$, the graininess function $\mu: \mathbb{T} \rightarrow[0, \infty)$ is defined by $\mu(t)=\sigma(t)-t$. The set $\mathbb{T}^{\kappa}$ is derived from the time scale $\mathbb{T}$ as follows: If $\mathbb{T}$ has a left-scattered maximum $\gamma$, then $\mathbb{T}^{\kappa}=\mathbb{T}-\{\gamma\}$. Otherwise, $\mathbb{T}^{\kappa}=\mathbb{T}$. Especially, given a time scale interval $[a, b]_{\mathbb{T}}=\{t \in \mathbb{T} \mid a \leq t \leq b\}$, if $\rho(b)=b$, then $[a, b]^{\kappa}=[a, b]_{\mathbb{T}}$. Otherwise, $[a, b]^{\kappa}=[a, b)_{\mathbb{T}}$. In essence, $[a, b)_{\mathbb{T}}=[a, \rho(b)]_{\mathbb{T}}$.

Let $g: \mathbb{T} \rightarrow \mathbb{R}$ be a real-valued function and let $t \in \mathbb{T}^{\kappa}$. Given any $\varepsilon>0$, if there exist a number $\alpha$ and a neighborhood $U$ of $t$ such that

$$
|g(\sigma(t))-g(s)-\alpha(\sigma(t)-s)| \leq \varepsilon|\sigma(t)-s|
$$


for all $s \in U$, then we say that $g$ is delta differentiable (or in short: $\Delta$-differentiable) at $t$. Correspondingly, the number $\alpha$ is called the $\Delta$-derivative and it is denoted by $g^{\Delta}(t)$. More generally, the function $g$ is said to be delta differentiable ( $\Delta$-differentiable) on $\mathbb{T}^{\kappa}$ provided the $\Delta$-derivative $g^{\Delta}(t)$ exists for all $t \in \mathbb{T}^{\kappa}$.

Definition 2.3 (Lupulescu [7]). Let $F: \mathbb{T} \rightarrow \mathcal{K}$ be an interval-valued function. Then we say that $F$ is $l$-nondecreasing (or $l$-nonincreasing) on $\mathbb{T}$ if the real-valued function $t \rightarrow \operatorname{len}(F(t))$ is nondecreasing (or nonincreasing) on $\mathbb{T}$. Generally, if $F$ is $l$-nondecreasing or $l$-nonincreasing on $\mathbb{T}$, then we say that $F$ is $l$-monotonic on $\mathbb{T}$.

Definition 2.4 (Lupulescu [7]). Let $F: \mathbb{T} \rightarrow \mathcal{K}$ be an interval-valued function and let $A \in \mathcal{K}$. If for every $\varepsilon>0$, there exists $\delta>0$ such that $\left\|F(t) \ominus_{g} A\right\|=d_{H}(F(t), A) \leq \varepsilon$ for all $t \in U_{\mathbb{T}}\left(t_{0}, \delta\right)$ (i.e., $\left.U_{\mathbb{T}}\left(t_{0}, \delta\right)=\left(t_{0}-\delta, t_{0}+\delta\right) \cap \mathbb{T}\right)$, then we say that $A$ is the $\mathbb{T}$-limit of $F$ at $t_{0} \in \mathbb{T}$. If $F$ has a $\mathbb{T}$-limit $A$ at $t_{0}$, then it is unique and is denoted by $A=\mathbb{T}-\lim _{t \rightarrow t_{0}} F(t)$.

An interval-valued function $F: \mathbb{T} \rightarrow \mathcal{K}$ is called $r d$-continuous if it is continuous at all right-dense points in $\mathbb{T}$ and its left-sided $\mathbb{T}$-limits exist at all left-dense points in $\mathbb{T}$.

Definition 2.5 (Lupulescu [7]). Let $F: \mathbb{T} \rightarrow \mathcal{K}$ be an interval-valued function and let $t \in \mathbb{T}^{\kappa}$. Then we define $F_{g H}^{\Delta}(t)$ to be the interval (provided it exists) with the property that for every $\varepsilon>0$, there exists $\delta>0$ such that

$$
d_{H}\left(F(\sigma(t)) \ominus_{g} F(s),(\sigma(t)-s) F_{g H}^{\Delta}(t)\right) \leq \varepsilon|\sigma(t)-s|
$$

for all $s \in U_{\mathbb{T}}(t, \delta)$. Here, $F_{g H}^{\Delta}(t)$ is called the delta generalized Hukuhara derivative $\left(\Delta_{g H^{-}}\right.$ derivative for short) of $F$ at $t$. Meantime, if $F_{g H}^{\Delta}(t)$ exists for each $t \in \mathbb{T}^{\kappa}$, then we say that $F$ is delta generalized Hukuhara differentiable $\left(\Delta_{g H}\right.$-differentiable for short) on $\mathbb{T}^{\kappa}$. In particular, the $\Delta_{g H}$-derivative $F_{g H}^{\Delta}$ degenerates into the $g H$-derivative $F_{g H}^{\prime}$ if the time scale $\mathbb{T}=\mathbb{R}$.

Theorem 2.6 (Lupulescu [7]). Assume that $F: \mathbb{T} \rightarrow \mathcal{K}$ is an interval-valued function and let $t \in \mathbb{T}^{\kappa}$. Then, the following statements are true:

(i) If $F: \mathbb{T} \rightarrow \mathcal{K}$ is $\Delta_{g H}$-differentiable at $t \in \mathbb{T}^{\kappa}$, then it is continuous at $t$;

(ii) If $F$ is continuous at $t$ and $t$ is right-scattered, then $F$ is $\Delta_{g H}$-differentiable at $t$ with

$$
F_{g H}^{\Delta}(t)=\frac{F(\sigma(t)) \ominus_{g} F(t)}{\mu(t)}
$$

(iii) If $t$ is right-dense, then $F$ is $\Delta_{g H}$-differentiable at $t$ if and only if the $\mathbb{T}$-limit

$$
\mathbb{T}-\lim _{s \rightarrow t} \frac{F(t) \ominus_{g} F(s)}{t-s}
$$

exists as a closed interval. In this case

$$
F_{g H}^{\Delta}(t)=\mathbb{T}-\lim _{s \rightarrow t} \frac{F(t) \ominus_{g} F(s)}{t-s}
$$

(iv) If $F$ is $\Delta_{g H}$-differentiable at $t$, then

$$
F(\sigma(t)) \ominus_{g} F(t)=\mu(t) F_{g H}^{\Delta}(t) .
$$

Finally, the induction principle on time scales is provided, which is useful in the next section.

Theorem 2.7 (Bohner and Peterson [7]). Let $t_{0} \in \mathbb{T}$ and let $\left\{S(t): t \in\left[t_{0},+\infty\right)\right\}$ be a family of statements satisfying:

(I) $S\left(t_{0}\right)$ is true;

(II) If $t \in\left[t_{0},+\infty\right)$ is right-scattered and $S(t)$ is true, then $S(\sigma(t))$ is also true; 
(III) If $t \in\left[t_{0},+\infty\right)$ is right-dense and $S(t)$ is true, then there is a neighborhood $U$ of $t$ such that $S(s)$ is true for all $s \in U \cap(t,+\infty)$;

(IV) If $t \in\left(t_{0},+\infty\right)$ is left-dense and $S(s)$ is true for all $s \in\left[t_{0}, t\right)$, then $S(t)$ is true. Then $S(t)$ is true for all $t \in\left[t_{0},+\infty\right)$.

\section{Mean value theorem for interval-valued functions on time scales}

Based on the works of Markov [8] and Lupulescu [7], in this section, we shall establish another version of the mean value theorem for interval-valued functions on time scales.

Theorem 3.1 (Markov [8]). Let $F$ be a continuous interval-valued function on $[a, b]$ and $g H$-differentiable in $(a, b)$. Then

$$
F(b) \ominus_{g} F(a) \subset(b-a) F_{g H}^{\prime}([a, b]),
$$

where $F_{g H}^{\prime}([a, b])=\bigcup_{\xi \in[a, b]} F_{g H}^{\prime}(\xi)$.

Remark 3.2. In general, it is not true that there exists $\xi \in[a, b]$ such that $F(b) \ominus_{g} F(a) \subset$ $(b-a) F_{g H}^{\prime}(\xi)$.

Theorem 3.3 (Lupulescu [7]). Let $F$ be a continuous and l-monotonic interval-valued function on $[a, b]_{\mathbb{T}}$ and let $F$ be $\Delta_{g H}$-differentiable in $[a, b)_{\mathbb{T}}$. Then

$$
F(b) \ominus_{g} F(a) \subset(b-a) F_{g H}^{\Delta}\left([a, b)_{\mathbb{T}}\right),
$$

where $F_{g H}^{\Delta}\left([a, b)_{\mathbb{T}}\right)=\bigcup_{\xi \in[a, b)_{\mathbb{T}}} F_{g H}^{\Delta}(\xi)$.

Theorem 3.4. Let $F$ and $g$ be an interval-valued function and a real-valued function defined on $\mathbb{T}$, respectively. Assume that $F$ is $\Delta_{g H}$-differentiable and $g$ is $\Delta$-differentiable on $\mathbb{T}^{\kappa}$. If

$$
\left\|F_{g H}^{\Delta}(t)\right\| \leq g^{\Delta}(t)
$$

for all $t \in \mathbb{T}^{\kappa}$, then

$$
\left\|F(t) \ominus_{g} F(r)\right\| \leq g(t)-g(r)
$$

for all $t \in[r, s]_{\mathbb{T}}$ with $r, s \in \mathbb{T}$ and $r \leq s$.

Proof. Let $r, s \in \mathbb{T}$ with $r \leq s$. For any $\varepsilon>0$, we can show by the induction principle as shown in Theorem 2.7 that

$$
S(t):\left\|F(t) \ominus_{g} F(r)\right\| \leq g(t)-g(r)+\varepsilon(t-r)
$$

holds for all $t \in[r, s]_{\mathbb{T}}$. The proof is divided into four steps.

(I) If $t=r$, then the statement $S(r)$ is obviously true.

(II) Assume that $t$ is right-scattered and $S(t)$ is satisfied. According to Definition 2.1 and Theorem 2.6 (iv), we have the following two cases:

Case (a):

$$
\begin{aligned}
\left\|F(\sigma(t)) \ominus_{g} F(r)\right\| & =d_{H}(F(\sigma(t)), F(r)) \\
& =d_{H}\left(F(t)+\mu(t) F_{g H}^{\Delta}(t), F(r)\right) \\
& \leq d_{H}(F(t), F(r))+d_{H}\left(\mu(t) F_{g H}^{\Delta}(t),\{0\}\right) \\
& =d_{H}(F(t), F(r))+\mu(t) d_{H}\left(F_{g H}^{\Delta}(t),\{0\}\right) \\
& =d_{H}(F(t), F(r))+\mu(t)\left\|F_{g H}^{\Delta}(t)\right\| \\
& \leq d_{H}(F(t), F(r))+\mu(t) g^{\Delta}(t) \\
& \leq g(t)-g(r)+\varepsilon(t-r)+g(\sigma(t))-g(t) \\
& =g(\sigma(t))-g(r)+\varepsilon(t-r) \\
& \leq g(\sigma(t))-g(r)+\varepsilon(\sigma(t)-r) .
\end{aligned}
$$


Case (b):

$$
\begin{aligned}
\left\|F(\sigma(t)) \ominus_{g} F(r)\right\| & =d_{H}(F(\sigma(t)), F(r)) \\
& =d_{H}\left(F(\sigma(t))+(-1) \mu(t) F_{g H}^{\Delta}(t), F(r)+(-1) \mu(t) F_{g H}^{\Delta}(t)\right) \\
& =d_{H}\left(F(t), F(r)+(-1) \mu(t) F_{g H}^{\Delta}(t)\right) \\
& \leq d_{H}(F(t), F(r))+d_{H}\left(\{0\},(-1) \mu(t) F_{g H}^{\Delta}(t)\right) \\
& =d_{H}(F(t), F(r))+\mu(t) d_{H}\left(\{0\}, F_{g H}^{\Delta}(t)\right) \\
& =d_{H}(F(t), F(r))+\mu(t)\left\|F_{g H}^{\Delta}(t)\right\| \\
& \leq g(\sigma(t))-g(r)+\varepsilon(\sigma(t)-r) .
\end{aligned}
$$

Thus, the statement $S(\sigma(t))$ is satisfied.

(III) Suppose that $S(t)$ holds and $t \neq s$ is right-dense. Clearly, $\sigma(t)=t$. Since $F$ is

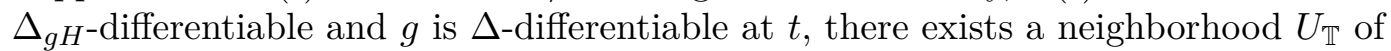
$t$ such that

$$
d_{H}\left(F(t) \ominus_{g} F(s), F_{g H}^{\Delta}(t)(t-s)\right) \leq \frac{\varepsilon}{2}|t-s|
$$

for all $s \in U_{\mathbb{T}}$ and

$$
\left|g(t)-g(s)-g^{\Delta}(t)(t-s)\right| \leq \frac{\varepsilon}{2}|t-s|
$$

for all $s \in U_{\mathbb{T}}$. Therefore, we can obtain that

$$
\begin{aligned}
d_{H}(F(t), F(s)) & =d_{H}\left(F(t) \ominus_{g} F(s),\{0\}\right) \\
& \leq d_{H}\left(F(t) \ominus_{g} F(s), F_{g H}^{\Delta}(t)(t-s)\right)+d_{H}\left(\{0\}, F_{g H}^{\Delta}(t)(t-s)\right) \\
& \leq\left(\left\|F_{g H}^{\Delta}(t)\right\|+\frac{\varepsilon}{2}\right)|t-s|
\end{aligned}
$$

and

$$
g(s)-g(t)-g^{\Delta}(t)(s-t) \geq-\frac{\varepsilon}{2}|t-s|
$$

for all $s \in U_{\mathbb{T}}$. Hence, for all $s \in U_{\mathbb{T}} \cap(t, \infty)$, we have

$$
\begin{aligned}
\left\|F(s) \ominus_{g} F(r)\right\| & =d_{H}(F(s), F(r)) \\
& \leq d_{H}(F(s), F(t))+d_{H}(F(t), F(r)) \\
& \leq\left(\left\|F_{g H}^{\Delta}(t)\right\|+\frac{\varepsilon}{2}\right)|t-s|+d_{H}(F(t), F(r)) \\
& \leq\left(g^{\Delta}(t)+\frac{\varepsilon}{2}\right)|t-s|+d_{H}(F(t), F(r)) \\
& \leq\left(g^{\Delta}(t)+\frac{\varepsilon}{2}\right)|t-s|+g(t)-g(r)+\varepsilon(t-r) \\
& =g^{\Delta}(t)(s-t)+\frac{\varepsilon}{2}(s-t)+g(t)-g(r)+\varepsilon(t-r) \\
& \leq g(s)-g(t)+\frac{\varepsilon}{2}|t-s|+\frac{\varepsilon}{2}(s-t)+g(t)-g(r)+\varepsilon(t-r) \\
& =g(s)-g(r)+\varepsilon(s-r),
\end{aligned}
$$

which implies that $S(s)$ holds for all $s \in U_{\mathbb{T}} \cap(t, \infty)$.

(IV) Let $t$ be left-dense and assume that $S(\tau)$ holds for all $\tau<t$. By the continuity of $F$ and $g$, we then obtain that

$$
\begin{aligned}
\left\|F(t) \ominus_{g} F(r)\right\| & =\lim _{\tau \rightarrow t-}\left\|F(\tau) \ominus_{g} F(r)\right\| \\
& \leq \lim _{\tau \rightarrow t-} g(\tau)-g(r)+\varepsilon(\tau-r) \\
& =g(t)-g(r)+\varepsilon(t-r),
\end{aligned}
$$


which means that the statement $S(t)$ is true.

Due to the arbitrariness of $\varepsilon$, we have obtained the desired result and completed the proof of this theorem.

As an application of Theorem 3.4, we can obtain the following results.

Corollary 3.5. Let $F, G: \mathbb{T} \rightarrow \mathcal{K}$ be two $\Delta_{g H}$-differentiable interval-valued functions on $\mathbb{T}^{\kappa}$. Then

(i) If $D$ is a compact interval with endpoints $r, s \in \mathbb{T}$, then

$$
\left\|F(s) \ominus_{g} F(r)\right\| \leq\left(\sup _{t \in D^{\kappa}}\left\|F_{g H}^{\Delta}(t)\right\|\right)|s-r| .
$$

(ii) If $F_{g H}^{\Delta}(t)=\{0\}$ for all $t \in \mathbb{T}^{\kappa}$, then $F$ is a constant interval.

(ii) If both $F$ and $G$ are l-nondecreasing or l-nonincreasing, and $F_{g H}^{\Delta}(t)=G_{g H}^{\Delta}(t)$ for all $t \in \mathbb{T}^{\kappa}$, then

$$
F(t) \ominus_{g} G(t)=C
$$

for all $t \in \mathbb{T}$, where $C$ is a constant interval.

(iv) If $F$ and $G$ are such that one is l-nondecreasing and the other is l-nonincreasing, and $F_{g H}^{\Delta}(t)=-G_{g H}^{\Delta}(t)$ for all $t \in \mathbb{T}^{\kappa}$, then

$$
F(t)+G(t)=C
$$

for all $t \in \mathbb{T}$, where $C$ is a constant interval.

Proof. (i) Let $r, s \in \mathbb{T}$ with $r \leq s$. Define

$$
g(t):=\left(\sup _{\tau \in[r, s]^{\kappa}}\left\|F_{g H}^{\Delta}(\tau)\right\|\right)(t-r)
$$

for $t \in \mathbb{T}$. Then, it is easy to know that

$$
g^{\Delta}(t)=\sup _{\tau \in[r, s]^{\kappa}}\left\|F_{g H}^{\Delta}(\tau)\right\| \geq\left\|F_{g H}^{\Delta}(t)\right\|
$$

for all $\tau \in[r, s]^{\kappa}$. By Theorem 3.4, the desired result can be obtained.

(ii) It is a direct consequence of part (i).

(iii) By Theorem 4 in [7], we have

$$
\left(F(t) \ominus_{g} G(t)\right)_{g H}^{\Delta}=F_{g H}^{\Delta}(t) \ominus_{g} G_{g H}^{\Delta}(t)=F_{g H}^{\Delta}(t) \ominus_{g} F_{g H}^{\Delta}(t)=\{0\}
$$

for $t \in \mathbb{T}^{\kappa}$. The desired result follows immediately from (ii).

(iv) Similar to part (iii), since

$$
(F(t)+G(t))_{g H}^{\Delta}=F^{\Delta}(t) \ominus_{g}\left(-G_{g H}^{\Delta}(t)\right)=F_{g H}^{\Delta}(t) \ominus_{g} F_{g H}^{\Delta}(t)=\{0\}
$$

for $t \in \mathbb{T}^{\kappa}$.

Remark 3.6. If $F$ and $G$ are differently $l$-monotonic in (iii) of Corollary 3.5, in general, there is no constant interval $C$ such that $F(t) \ominus_{g} G(t)=C$. Analogously, $F$ and $G$ are equally $l$-monotonic in (iv), then the result is not necessarily true.

Remark 3.7. The results (iii) and (iv) of Corollary 3.4 coincide with Corollary 2 in [7].

Example 3.8. (i) Let $\mathbb{T}=[0,1]$ and let $F(t)=[t, 2 t]$ and $G(t)=[2 t-1, t]$. Note that $\operatorname{len}(F(t))=t$ is nondecreasing on $\mathbb{T}$ and $\operatorname{len}(G(t))=1-t$ is nonincreasing on $\mathbb{T}$. It is easy to check that $F(t)$ and $G(t)$ are $\Delta_{g H^{-}}$-differentiable on $\mathbb{T}^{\kappa}=[0,1]$ and $F_{g H}^{\Delta}(t)=F_{g H}^{\prime}(t)=$ $[1,2]=G_{g H}^{\prime}(t)=G_{g H}^{\Delta}(t)$ for each $t \in[0,1]$ (Only consider the unilateral derivative at the endpoints 0 and 1). However, there is no constant interval $C$ such that $F(t) \ominus_{g} G(t)=C$.

(ii) Let $\mathbb{T}=[0,1]$ and let $F(t)=[-t, 2 t]$ and $G(t)=[t-1,2(1-t)]$. Clearly, len $(F(t))=$ $3 t$ is nondecreasing and $\operatorname{len}(G(t))=3(1-t)$ in nonincreasing on $\mathbb{T}$. It can easily be verified that $F(t)$ and $G(t)$ are $\Delta_{g H}$-differentiable on $\mathbb{T}^{\kappa}=[0,1]$. Moreover, $F_{g H}^{\Delta}(t)=F_{g H}^{\prime}(t)=$ 
$[-1,2], G_{g H}^{\Delta}(t)=G_{g H}^{\prime}(t)=[-2,1]$ for each $t \in[0,1]$. Then, we have $F_{g H}^{\Delta}(t)=-G_{g H}^{\Delta}(t)$ for each $t \in[0,1]$. By Corollary 3.5, there exists an interval $C=[-1,2]$ such that $F(t)+G(t)=[-1,2]=C$.

Example 3.9. Let $\mathbb{T}=h \mathbb{Z}_{0}^{+}=\left\{h k: k \in \mathbb{Z}_{0}^{+}\right\}, h>0$. Suppose $F(t)=\left[t, t^{2}\right]$ and $G(t)=\left[t+a, t^{2}+b\right]$, where $a$ and $b$ are two fixed constants with $a \leq b$. Obviously, both len $(F(t))=t(t-1)$ and $\operatorname{len}(G(t))=t(t-1)+b-a$ are $l$-nondecreasing on $\mathbb{T}$. By Theorem 2.6 , we can obtain $F_{g H}^{\Delta}(t)=[1,2 t]=G_{g H}^{\Delta}(t)$ for each $t \in \mathbb{T}$. Therefore, we can find an interval $C=[-b,-a]$ such that $F(t) \ominus_{g} G(t)=C$ on $\mathbb{T}$.

Example 3.10. Let $\mathbb{T}=\mathbb{R}$ and let $F(t)=\left[-2 e^{-t}-1, e^{-t}+2\right]$ and $G(t)=\left[-2 e^{-t}, e^{-t}+1\right]$. Obviously, $\operatorname{len}(F(t))=3+3 e^{-t}$ and $\operatorname{len}(G(t))=1+3 e^{-t}$ are nonincreasing on $\mathbb{R}$. It is

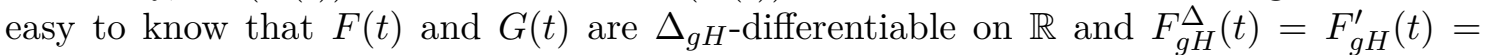
$[-1,2] e^{-t}=G_{g H}^{\prime}(t)=G_{g H}^{\Delta}(t)$ for each $t \in \mathbb{R}$. By Corollary 3.5, we can find an interval $C=[-1,1]$ such that $F(t) \ominus_{g} G(t)=C$.

Example 3.11. Let $\mathbb{T}=q^{\mathbb{Z}}=\left\{q^{k} \mid k \in \mathbb{Z}\right\}$, where $q>1$. Assume $F(t)=\left[-t, 2 t^{2}\right]$ and $G(t)=\left[-2 t^{2}+1, t+2\right]$. According to Theorem 2.6, for each $t \in \mathbb{T}$, it follows that

$$
\begin{aligned}
F_{g H}^{\Delta}(t) & =\frac{F(\sigma(t)) \ominus_{g} F(t)}{\mu(t)} \\
& =\frac{F(q t) \ominus_{g} F(t)}{(q-1) t} \\
& =\frac{\left[-q t, 2 q^{2} t^{2}\right] \ominus_{g}\left[-t, 2 t^{2}\right]}{(q-1) t} \\
& =\frac{\left[-(q-1) t, 2\left(q^{2}-1\right) t^{2}\right]}{(q-1) t} \\
& =[-1,2(q+1) t] .
\end{aligned}
$$

Using the similar method, we can obtain $G_{g H}^{\Delta}(t)=[-2(q+1) t, 1]=-F_{g H}^{\Delta}(t)$. However, $\operatorname{len}(F(t))=2 t^{2}+t$ and $\operatorname{len}(G(t))=2 t^{2}+t+1$ are nondecreasing on $\mathbb{T}$. Therefore, the conditions of Corollary 3.5 are not satisfied. Indeed, there does not exist an interval $C$ such that $F(t)+G(t)=C$.

Example 3.12. Let $\mathbb{T}=\mathbb{N}_{0}^{2}=\left\{n^{2} \mid n \in \mathbb{N}_{0}\right\}$ and let $F(t)=[-\sqrt{t}, \sqrt{t}]$ and $G(t)=$ $[\min \{1-\sqrt{t}, \sqrt{t}\}, \max \{1-\sqrt{t}, \sqrt{t}\}]$. For every $t \in \mathbb{T}$, it is easy to know that $t$ is rightscattered. By Theorem 2.6, we can obtain

$$
\begin{aligned}
F_{g H}^{\Delta}(t) & =\frac{F(\sigma(t)) \ominus_{g} F(t)}{\mu(t)} \\
& =\frac{F\left((\sqrt{t}+1)^{2}\right) \ominus_{g} F(t)}{2 \sqrt{t}+1} \\
& =\frac{[-\sqrt{t}-1, \sqrt{t}+1] \ominus_{g}[-\sqrt{t}, \sqrt{t}]}{2 \sqrt{t}+1} \\
& =\frac{1}{2 \sqrt{t}+1}[-1,1] .
\end{aligned}
$$

Similarly, we can infer that $G_{g H}^{\Delta}(t)=\frac{1}{2 \sqrt{t}+1}[-1,1]=F_{g H}^{\Delta}(t)$. Although $\operatorname{len}(F(t))=2 \sqrt{t}$ is nondecreasing on $\mathbb{T}$, len $(G(t))=|2 \sqrt{t}-1|$ is not monotonic on $\mathbb{T}$. Therefore, the conditions of Corollary 3.5 are not satisfied. In fact, there does not exist an interval $C$ such that $F(t) \ominus_{g} G(t)=C$. 


\section{4. $C_{0}$-Semigroups for interval-valued functions on time scales}

In this section, we shall introduce some basic notions and results associated with semigroups of operators for interval-valued functions on time scales.

Definition 4.1. Let $\widetilde{A}: \mathcal{K} \rightarrow \mathcal{K}$. $\widetilde{A}$ is said to be a linear operator on $\mathcal{K}$ if

$$
\widetilde{A}(\alpha \cdot x+\beta \cdot y)=\alpha \cdot \widetilde{A}(x)+\beta \cdot \widetilde{A}(y)
$$

for all $x, y \in \mathcal{K}$ and $\alpha, \beta \in \mathbb{R}$.

Remark 4.2. Unlike the property of linear operators on a linear space, it should be noticed that the continuity of a linear operator $\widetilde{A}$ at $\{0\} \in \mathcal{K}$ does not imply the continuity of $\widetilde{A}$ at each $x \in \mathcal{K}$, because $(\mathcal{K},+, \cdot)$ is not a linear space, in general, the equality $x_{0}=\left(x_{0} \ominus_{g} x\right)+x$ does not hold, unless $\operatorname{len}\left(x_{0}\right) \geq \operatorname{len}(x)$.

Lemma 4.3. Let $\widetilde{A}$ be a linear operator on $\mathcal{K}$. Then, for all $x, y \in \mathcal{K}$, we have

$$
\widetilde{A}\left(x \ominus_{g} y\right)=\widetilde{A}(x) \ominus_{g} \widetilde{A}(y) .
$$

Proof. Let $z=x \ominus_{g} y$. Then, we get $x=y+z$ or $y=x+(-z)$. According to Definition 4.1 , it follows that

$$
\left\{\begin{array}{l}
\widetilde{A}(x)=\widetilde{A}(y+z)=\widetilde{A}(y)+\widetilde{A}(z), \\
\text { or } \widetilde{A}(y)=\widetilde{A}(x+(-z))=\widetilde{A}(x)+\widetilde{A}(-z),
\end{array}\right.
$$

which is equivalent to

$$
\left\{\begin{array}{l}
\widetilde{A}(x)=\widetilde{A}(y)+\widetilde{A}(z), \\
\text { or } \widetilde{A}(y)=\widetilde{A}(x)+(-1) \widetilde{A}(z) .
\end{array}\right.
$$

Therefore, $\widetilde{A}\left(x \ominus_{g} y\right)=\widetilde{A}(z)=\widetilde{A}(x) \ominus_{g} \widetilde{A}(y)$.

$$
L(\mathcal{K})=\{\widetilde{A}: \mathcal{K} \rightarrow \mathcal{K} \mid \widetilde{A} \text { is linear and continuous at each } x \in \mathcal{K}\} .
$$

Let us introduce the addition and scalar multiplication in $L(\mathcal{K})$ as follows

$$
(\widetilde{A}+\widetilde{B})(x)=\widetilde{A}(x)+\widetilde{B}(x), \quad(\lambda \cdot \widetilde{A})(x)=\lambda \cdot \widetilde{A}(x),
$$

for $\widetilde{A}, \widetilde{B} \in L(\mathcal{K})$ and $\lambda \in \mathbb{R}$. Consider the metric $D_{H}: L(\mathcal{K}) \times L(\mathcal{K}) \rightarrow[0,+\infty)$ defined by

$$
D_{H}(\widetilde{A}, \widetilde{B})=\sup \left\{d_{H}(\widetilde{A}(x), \widetilde{B}(x)):\|x\| \leq 1\right\},
$$

where $\|x\|=d_{H}(x, 0)$. From the properties of $d_{H}$, it can easily be verified that

(i) $D_{H}(\widetilde{A}+\widetilde{B}, \widetilde{C}+\widetilde{D}) \leq D_{H}(\widetilde{A}, \widetilde{C})+D_{H}(\widetilde{B}, \widetilde{D})$;

(ii) $D_{H}(\lambda \cdot \widetilde{A}, \lambda \cdot \widetilde{B})=|\lambda| D_{H}(\widetilde{A}, \widetilde{B})$;

(iii) $D_{H}(\widetilde{A}, \widetilde{B}) \leq D_{H}(\widetilde{A}, 0)+D_{H}(0, \widetilde{B})=\|\widetilde{A}\|+\|\widetilde{B}\|$;

(iv) $D_{H}(\widetilde{A}+\widetilde{B}, \widetilde{C}) \leq D_{H}(\widetilde{A}, \widetilde{C})+D_{H}(\widetilde{B}, \widetilde{C})$,

where $\widetilde{A}, \widetilde{B}, \widetilde{C}, \widetilde{D} \in L(\mathcal{K})$ and $\lambda \in \mathbb{R}$.

As a special case of Corollary 3.6 in [4], it is easy to know that $\left(L(\mathcal{K}), D_{H}\right)$ is a complete metric space.

Definition 4.4. Let $\mathbb{T} \subseteq \mathbb{R}_{0}^{+}$be a semigroup time scale. A $C_{0}$-semigroup $T$ on $\mathcal{K}$ is a family of continuous linear operators $\{T(t): t \in \mathbb{T}\} \subset L(\mathcal{K})$, which satisfies

(i) $T(0)=I, I$ is the identity operator on $\mathcal{K}$;

(ii) $T(t+s)=T(t) T(s)$ for every $t, s \in \mathbb{T}$;

(iii) $\lim _{t \rightarrow 0+} T(t) x=x$ for each $x \in \mathcal{K}$, i.e., $T(\cdot) x: \mathbb{T} \rightarrow \mathcal{K}$ is continuous at 0 . 
Definition 4.5. Let $T$ be a $C_{0}$-semigroup on $\mathcal{K}$. A linear operator $\widetilde{A}$ is called the generator of the $C_{0}$-semigroup $T$ if for all $x \in \mathcal{K}$, the limit

$$
\lim _{s \rightarrow 0+} \frac{T(\mu(t)) x \ominus_{g} T(s) x}{\mu(t)-s}=\widetilde{A} x
$$

exists uniformly in $t$. Here the limit are considered in the metric $d_{H}$.

Example 4.6. Let $\mathbb{T}=h \mathbb{Z}_{0}^{+}=\left\{h k: k \in \mathbb{Z}_{0}^{+}\right\}, h>0$ and $\widetilde{A}$ be a continuous linear operator on $\mathcal{K}$. Then $\widetilde{A}$ is the generator of $T(t)=(I+t \widetilde{A})^{t / h}$ for $t \in h \mathbb{Z}_{0}^{+}$. In fact, for $x \in \mathcal{K}$, we have

$$
\begin{aligned}
& \lim _{s \rightarrow 0+} \frac{T(\mu(t)) x \ominus_{g} T(s) x}{\mu(t)-s} \\
& =\lim _{s \rightarrow 0+} \frac{T(h) x \ominus_{g} T(s) x}{h-s} \\
& =\frac{T(h) x \ominus_{g} I x}{h} \\
& =\frac{(I+h \widetilde{A}) x \ominus_{g} x}{h}=\widetilde{A} x .
\end{aligned}
$$

Lemma 4.7. Let $\widetilde{A} \in L(\mathcal{K})$ and $\widetilde{A}^{0}=I, \widetilde{A}^{k+1}=\widetilde{A}^{k} \widetilde{A}, k=0,1,2, \ldots$ Then the sequence of operators $\left\{S_{n}(t)\right\}, t \in \mathbb{R}_{0}^{+}$, is a Cauchy sequence in $L(\mathcal{K})$, where $S_{n}(t)=\sum_{k=0}^{n} \frac{t^{k}}{k !} \cdot \widetilde{A}^{k}$.

Proof. It is a direct consequence of Theorem 3.9 in [4].

In view of the completeness of $L(\mathcal{K})$ and Lemma 4.3, there exists $T(t) \in L(\mathcal{K})$ such that the sequence of operators $\left\{S_{n}(t)\right\}$ converges to $T(t)$ for each $t \in \mathbb{R}_{0}^{+}$. Formally, we denote $T(t)$ by

$$
e^{t \cdot \widetilde{A}} \triangleq \sum_{k=0}^{\infty} \frac{t^{k}}{k !} \cdot \widetilde{A}^{k}
$$

Lemma 4.8. Let $\mathbb{T}=\mathbb{R}_{0}^{+}$and $\widetilde{A} \in L(\mathcal{K})$. Define $T(t)=e^{t \cdot \widetilde{A}}, t \in \mathbb{T}$, then

(i) $T(t+s)=T(t) T(s)$ for all $t, s \in \mathbb{T}$;

(ii) $\lim _{s \rightarrow 0+} \frac{T(s) x \ominus_{g} x}{s}=\widetilde{A} x$ for each $x \in \mathcal{K}$.

Proof. (i) By Theorem 3.9 (ii) in [4], it is obvious.

(ii) According to Proposition 5 in [7], this result can be proved in a similar way as in Theorem 3.9 in [4].

Example 4.9. Let $\mathbb{T}=\mathbb{R}_{0}^{+}$and $\widetilde{A} \in L(\mathcal{K})$. Then $\widetilde{A}$ is the generator of $T(t)=e^{t \cdot \widetilde{A}}$ for $t \in \mathbb{R}_{0}^{+}$. In fact, by Lemma 4.8 , for $x \in \mathcal{K}$, we have

$$
\begin{aligned}
& \lim _{s \rightarrow 0+} \frac{T(\mu(t)) x \ominus_{g} T(s) x}{\mu(t)-s} \\
& =\lim _{s \rightarrow 0+} \frac{T(s) x \ominus_{g} T(0) x}{s} \\
& =\widetilde{A} x .
\end{aligned}
$$

Lemma 4.10. Let $\mathbb{T} \subseteq \mathbb{R}_{0}^{+}$be a semigroup time scale. Then for each $x \in \mathcal{K}$, the function $T(\cdot) x: t \mapsto T(t) x$ is continuous from $\mathbb{T}$ into $\mathcal{K}$. 
Proof. Let $t \in \mathbb{T}$. For all $0<s \in \mathbb{T}$, we get

$$
\begin{aligned}
d_{H}(T(t+s) x, T(t) x) & =d_{H}\left(T(t+s) x \ominus_{g} T(t) x, 0\right) \\
& =d_{H}\left(T(t) T(s) x \ominus_{g} T(t) x, 0\right) \\
& =\left\|T(t)\left(T(s) x \ominus_{g} x\right)\right\| \\
& \leq\|T(t)\|\left\|T(s) x \ominus_{g} x\right\| .
\end{aligned}
$$

Letting $s \rightarrow 0+,\left\|T(s) x \ominus_{g} x\right\| \rightarrow 0$, which implies the continuity of $T(t) x$ at $t \in \mathbb{T}$.

Theorem 4.11. Let $\mathbb{T} \subseteq \mathbb{R}_{0}^{+}$be a semigroup time scale with the constant graininess function $\mu(t)=h$. Suppose that $T$ is a $C_{0}$-semigroup on $\mathcal{K}$. Then $T(t)$ is $\Delta_{g H}$-differentiable in $t \in \mathbb{T}$, and

$$
T_{g H}^{\Delta}(t)=\widetilde{A}[T(t)]
$$

Proof. (i) If $\mu(t)=h>0$, then $t$ is right-scattered. By Lemma 2.3 in [5], we know $T=h \mathbb{Z}_{0}^{+}$. Furthermore, according to Lemma 4.4, $T(t)$ is continuous at $t$, so $T(t)$ is $\Delta_{g H}$-differentiable. From Example 4.6, we can obtain

$$
\begin{aligned}
T_{g H}^{\Delta}(t) & =\frac{T(\sigma(t)) \ominus_{g} T(t)}{\mu(t)} \\
& =\frac{T(t+h) \ominus_{g} T(t)}{h} \\
& =\frac{T(h) T(t) \ominus_{g} T(t)}{h} \\
& =\widetilde{A}[T(t)] .
\end{aligned}
$$

(ii) If $\mu(t)=h=0$, then $t$ is right-dense. In view of Lemma 2.3 in [5], $T=\mathbb{R}_{0}^{+}$. Based on Lemma 4.8 , we can obtain the above result by using a similar argument as in Theorem 3.9 (iv) in [4].

Definition 4.12. Let $\mathbb{T} \subseteq \mathbb{R}_{0}^{+}$be a semigroup time scale and let $T$ be a $C_{0}$-semigroup on $\mathcal{K}$. We say that $T$ is a $l$-monotonic $C_{0}$-semigroup on $\mathcal{K}$ if the interval-valued function $T(\cdot) x: \mathbb{T} \rightarrow \mathcal{K}$ is $l$-monotonic for every $x \in \mathcal{K}$.

Lemma 4.13. Let $\mathbb{T} \subseteq \mathbb{R}_{0}^{+}$be a semigroup time scale and let $T$ be a $C_{0}$-semigroup on $\mathcal{K}$. Assume that $g: \mathbb{T} \rightarrow \mathcal{K}$ is rd-continuous on $\mathbb{T}$. Define $F(t)=\int_{0}^{t} T(t-s) g(s) \Delta s$. If $T$ is $l$-nondecreasing on $\mathcal{K}$, then $F$ is also l-nondecreasing on $\mathcal{K}$.

Proof. Let $t_{1}, t_{2} \in \mathbb{T}$ with $t_{1}<t_{2}$. Then, we have

$$
\begin{aligned}
T\left(t_{2}-t_{1}\right) F\left(t_{1}\right) & =\int_{0}^{t_{1}} T\left(t_{2}-s\right) g(s) \Delta s \\
& \subseteq \int_{0}^{t_{1}} T\left(t_{2}-s\right) g(s) \Delta s+\int_{t_{1}}^{t_{2}} T\left(t_{2}-s\right) g(s) \Delta s \\
& =\int_{0}^{t_{2}} T\left(t_{2}-s\right) g(s) \Delta s=F\left(t_{2}\right) .
\end{aligned}
$$

Since $T$ is $l$-nondecreasing on $\mathcal{K}$, it follows that

$$
F\left(t_{1}\right) \subseteq T\left(t_{2}-t_{1}\right) F\left(t_{1}\right) \subseteq F\left(t_{2}\right),
$$

which implies $\operatorname{len}\left(F\left(t_{1}\right)\right) \leq \operatorname{len}\left(F\left(t_{2}\right)\right)$. Namely, $F$ is $l$-nondecreasing on $\mathcal{K}$.

Theorem 4.14. Let $\mathbb{T} \subseteq \mathbb{R}_{0}^{+}$be a semigroup time scale with the constant graininess function $\mu(t)=h$. Assume that $x_{0} \in \mathcal{K}$ and $g: \mathbb{T} \rightarrow \mathcal{K}$ is rd-continuous on $\mathbb{T}$. If $T$ is a $l$-nondecreasing $C_{0}$-semigroup on $\mathcal{K}$, then

$$
x(t)=T(t)\left(x_{0}\right)+\int_{0}^{t} T(t-s) g(s) \Delta s
$$


is $\Delta_{g H^{-}}$-differentiable on $\mathbb{T}^{\kappa}$. And then, $x(t)$ satisfies

$$
\left\{\begin{array}{l}
x_{g H}^{\Delta}(t)=\widetilde{A}[x(t)]+T(\mu(t))(g(t)), \quad t \in \mathbb{T}^{\kappa}, \\
x(0)=x_{0},
\end{array}\right.
$$

where the integral (including the integral in Lemma 4.13) for interval-valued functions defined on $[0, t)_{\mathbb{T}}$ is considered in the Riemann sense (the detailed definition can be seen in $[$ [7]).

Proof. For every $\mathbb{T}^{\kappa}$, we set

$$
F(t)=\int_{0}^{t} T(t-s) g(s) \Delta s .
$$

Since $T$ is a $l$-nondecreasing $C_{0}$-semigroup on $\mathcal{K}$, by Lemma 4.13 , it is easy to know that $F(t)$ is $l$-nondecreasing on $\mathbb{T}^{\kappa}$. Now, we distinguish two cases.

(i) If $t \in \mathbb{T}^{\kappa}$ is right-scattered, then we get

$$
\begin{aligned}
F(\sigma(t))=F(t+h) & =\int_{0}^{t+h} T(t-s+h) g(s) \Delta s \\
& =T(h)\left(\int_{0}^{t+h} T(t-s) g(s) \Delta s\right) \\
& =T(h)\left(F(t)+\int_{t}^{t+h} T(t-s) g(s) \Delta s\right) \\
& =T(h)(F(t))+T(h)\left(\int_{t}^{t+h} T(t-s) g(s) \Delta s\right) \\
& =T(h)(F(t))+T(h)(h T(0)(g(t))) \\
& =T(h)(F(t))+h T(h)((g(t)))
\end{aligned}
$$

By Lemma 2.2, it follows from (4.3), Theorems 2.6 and 4.11 that

$$
F_{g H}^{\Delta}(t)=\frac{F(t+h) \ominus_{g} F(t)}{h}=\widetilde{A}[F(t)]+T(h) g(t),
$$

since $F$ is $l$-nondecreasing. By Theorem 4.11, we know that $x(t)$ is $\Delta_{g H}$-differentiable. Furthermore, we can infer from (4.1), (4.4) and Theorem 4 in [7] that

$$
\begin{aligned}
x^{\Delta}(t) & =\left(T(t)\left(x_{0}\right)+\int_{0}^{t} T(t-s) g(s) \Delta s\right)_{g H}^{\Delta} \\
& =\left(T(t)\left(x_{0}\right)\right)_{g H}^{\Delta}+\left(\int_{0}^{t} T(t-s) g(s) \Delta s\right)_{g H}^{\Delta} \\
& =\widetilde{A}\left[T(t)\left(x_{0}\right)\right]+\widetilde{A}[F(t)]+T(h) g(t) \\
& =\widetilde{A}\left[T(t)\left(x_{0}\right)+F(t)\right]+T(h) g(t) \\
& =\widetilde{A}[x(t)]+T(\mu(t)) g(t),
\end{aligned}
$$

which means that $x(t)$ satisfies (4.2).

(ii) If $t \in \mathbb{T}^{\kappa}$ is right-dense, the proof is similar to Theorem 3.9 in [4] and so is omitted.

Remark 4.15. From Lemma 4.13, we know that $F$ is $l$-nondecreasing on $\mathbb{T}$ if $T$ is $l$ nondecreasing $C_{0}$-semigroup on $\mathcal{K}$. Apparently, a question that deserves further consideration is whether $F$ is $l$-monotonic on $\mathbb{T}$ if $T$ is $l$-nonincreasing $C_{0}$-semigroup on $\mathcal{K}$. Furthermore, if $F$ is $l$-monotonic on $\mathbb{T}$, then we can consider another question from Theorem 4.14. In detail, what is the solution to the initial value problem (4.2) when $T$ is $l$-nonincreasing $C_{0}$-semigroup on $\mathcal{K}$ ?

Acknowledgment. This work was supported by the National Natural Science Foundation of China (No. 11701425). 


\section{References}

[1] M. Bohner and A. Peterson, Dynamic Equations on Time Scales: An Introduction with Applications, Birkhäuser Boston Inc., Boston, MA, 2001.

[2] M. Bohner and A. Peterson, Advances in Dynamic Equations on Time Scales, Birkhäuser, Boston, 2003.

[3] Y. Chalco-Cano, A. Rufián-Lizana, H. Román-Flores and M.D. Jiménez-Gamero, Calculus for interval-valued functions using generalized Hukuhara derivative and applications, Fuzzy Sets Syst. 219, 49-67, 2013.

[4] C.G. Gal and S.G. Gal, Semigroups of operators on spaces of fuzzy-number-valued functions with applications to fuzzy differential equations, J. Fuzzy Math. 13, 647$682,2005$.

[5] A.E. Hamza and K.M. Oraby, Semigroups of operators and abstract dynamic equations on time scales, Appl. Math. Comput. 270, 334-348, 2013.

[6] S. Hilger, Ein Makettenkalkül mit Anwendung auf Zentrumsmannigfaltigkeiten, Ph.D. thesis, Universität Würzburg, 1988.

[7] V. Lupulescu, Hukuhara differentiability of interval-valued functions and interval differential equations on time scales, Inform. Sciences, 248, 50-67, 2013.

[8] S. Markov, Calculus for interval functions of a real variable, Computing, 22, 325-337, 1979.

[9] S. Markov, On the algebraic properties of convex bodies and some applications, J. Convex Anal. 7, 129-166, 2000.

[10] L. Stefanini, A generalization of Hukuhara difference and division for interval and fuzzy arithmetic, Fuzzy Sets Syst. 161, 1564-1584, 2010.

[11] L. Stefanini and B. Bede, Generalized Hukuhara differentiability of interval-valued functions and interval differential equations, Nonlinear Anal. 71, 1311-1328, 2009.

[12] J. Tao and Z. Zhang, Properties of interval-valued function space under the $g H$ difference and their application to semi-linear interval differential equations, Adv. Differ. Equ. 2016, Article number: 45, 2016. 